\title{
BMJ Open Influence of serum iron test results on the diagnosis of iron deficiency in children: a retrospective observational study
}

\author{
Gorkem Sezgin (D) , ${ }^{1}$ Ling Li, ${ }^{1}$ Johanna Westbrook, ${ }^{1}$ Elisabeth Wearne, ${ }^{2}$ \\ Denise Azar, ${ }^{2}$ Adam McLeod, ${ }^{3}$ Christopher Pearce, ${ }^{3}$ Vera Ignjatovic, ${ }^{4,5}$ \\ Paul Monagle, ${ }^{5,6}$ A Georgiou (iD ${ }^{1}$
}

To cite: Sezgin G, Li L, Westbrook J, et al. Influence of serum iron test results on the diagnosis of iron deficiency in children: a retrospective observational study. BMJ Open 2021;11:e046865. doi:10.1136/ bmjopen-2020-046865

- Prepublication history and additional supplemental material for this paper are available online. To view these files, please visit the journal online (http://dx.doi.org/10.1136/ bmjopen-2020-046865)

Received 12 November 2020 Accepted 08 June 2021
Check for updates

(C) Author(s) (or their employer(s)) 2021. Re-use permitted under CC BY-NC. No commercial re-use. See rights and permissions. Published by BMJ.

For numbered affiliations see end of article.

Correspondence to Mr Gorkem Sezgin; gorkem.sezgin@mq.edu.au

\section{ABSTRACT}

Background and objective Serum iron results are not indicative of iron deficiency yet may be incorrectly used to diagnose iron deficiency instead of serum ferritin results. Our objective was to determine the association between serum iron test results and iron-deficiency diagnosis in children by general practitioners.

Design, setting, patients and main outcome

measures A retrospective observational study of 14187 children aged 1-18 years with serum ferritin and serum iron test results from 137 general practices in Victoria, Australia, between 2008 and 2018. Generalised estimating equation models calculating 0 Rs were used to determine the association between serum iron test results (main exposure measure) and iron-deficiency diagnosis (outcome measure) in the following two population groups: (1) irondeplete population, defined as having a serum ferritin $<12 \mu \mathrm{g} / \mathrm{L}$ if aged $<5$ years and $<15 \mu \mathrm{g} / \mathrm{L}$ if aged $\geq 5$ years and (2) iron-replete population, defined as having a serum ferritin $>30 \mu \mathrm{g} / \mathrm{L}$.

Results 3484 tests were iron deplete and 15528 were iron replete. Iron-deplete children were less likely to be diagnosed with iron deficiency if they had normal serum iron levels (adjusted OR (AOR): $0.73 ; 95 \% \mathrm{Cl} 0.57$ to 0.96 ). Iron-replete children had greater odds of an iron-deficiency diagnosis if they had low serum iron results (AOR: 2.59; $95 \% \mathrm{Cl} 1.72$ to 3.89 ). Other contributors to an irondeficiency diagnosis were female sex and having anaemia Conclusion Serum ferritin alone remains the best means of diagnosing iron deficiency. Reliance on serum iron test results by general practitioners is leading to significant overdiagnosis and underdiagnosis of iron deficiency in children.

\section{INTRODUCTION}

Iron deficiency is the most common nutritional deficiency worldwide and the highest contributing factor to anaemia. The global prevalence of anaemia was estimated as $32.9 \%$ in 2010, with the highest burden among young children. ${ }^{1}$ Missed diagnosis of iron deficiency can be debilitating for young children, with studies suggesting a link between
Strengths and limitations of this study

- Our study uses general practice electronic health record data which contain a large sample size to draw our analysis from.

- Our study contains data from 137 general practices across a large region.

- Through general practice data, our study is able to correlate diagnostic procedures associated with pathology test orders, and to an extent, treatment thereafter.

- General practice data are limited to information recorded in the electronic health software, which is not always complete for all entry fields.

iron-deficiency anaemia and cognitive disorders. $^{2}$ Iron deficiency occurs some time before patients become anaemic, marked by a process in which red cells become microcytic and hypochromic. ${ }^{3}$ Therefore, anaemia can be prevented with early diagnosis and treatment of iron deficiency.

Diagnosing iron deficiency can be challenging: patients may present with nonspecific clinical symptoms and interpretation of laboratory tests in diagnosing iron deficiency can be difficult. ${ }^{4}$ Low serum ferritin levels (a measure of iron stores in the body) are clinically indicative of iron deficiency. ${ }^{5}$ In general practice, serum ferritin is often ordered as part of an iron panel test, which also contains serum iron, transferrin and total iron binding capacity. The provision of these accompanying tests can complicate the interpretation of results for diagnosing iron deficiency, particularly due to the presence of serum iron (a measure of circulating iron), which is not clinically indicative of iron deficiency. The utility of serum iron in clinical diagnosis is limited, particularly in general practice. Serum iron and transferrin 
saturation levels, when interpreted together, provide an indication of iron use by tissue. ${ }^{6}$ However, their use in diagnosing iron deficiency is not supported by current guidelines. ${ }^{6}$

Research focusing on diagnostic errors among paediatric patients is limited, particularly in general practice. Failure to order appropriate laboratory tests and incorrect interpretation of test results commonly go unrecognised, ${ }^{7}$ and are a considerable cause of diagnostic errors and adverse outcomes to patients. ${ }^{8}$ Our aim was to determine whether serum iron test results (which are often ordered alongside serum ferritin) were mistakenly used in the diagnosis of iron deficiency for paediatric patients in general practice. We, therefore, assessed the association between serum iron test results and the diagnosis of iron deficiency.

\section{METHODS}

We conducted a retrospective observational study of the association between serum iron test results for children, and their subsequent diagnosis of iron deficiency. We obtained our data from general practice electronic health records from 137 practices in Victoria, Australia. ${ }^{9}$ This study was conducted and reported according to Reporting of Studies Conducted Using Observational Routinely Collected Health Data Statement for Pharmacoepidemiology (RECORD-PE) guidelines. ${ }^{10}$

\section{Study population and variables}

Our sample population consisted of paediatric patients aged between 1 and 18 years with iron panel test results. We included laboratory tests containing both serum iron and serum ferritin test results between 2008 and 2018.

Serum ferritin, serum iron, transferrin saturation (TSAT), C reactive protein (CRP) tests and red blood cell indices were identified from laboratory test records by searching for related terms in Logical Observation Identifiers Names and Codes ${ }^{11}$ and test name fields. We determined ages by subtracting the years between test request date and patient year of birth.

Diagnostic records of iron deficiency were identified by searching Systematized Nomenclature of Medicine ${ }^{12}$ terms for 'iron' related to iron deficiency, and additionally, text fields for 'iron', 'ID' and 'IDA' related to iron deficiency. Specialist referrals were identified by searching for the terms 'referral' and 'specialist'. Medications used in the treatment of iron deficiency were identified by searching for commercial and generic names of iron supplements in the field record of medications prescribed by the general practitioner. Owing to limitations related to electronic health records, the association between test results and related diagnoses, referrals and medication is not recorded. The closest iron-deficiency diagnosis, referral and medication within a cut-off of 14 days after the test request was considered as being associated with the test result. Treatment for iron deficiency is recommended to be followed up with a repeat test within a 3-month period. We, therefore, identified test requests with a follow-up serum ferritin test within a 90-day period, which may indicate that the patient is being treated.

\section{Inclusion/exclusion criteria}

To ensure our study population contained only new cases, we excluded cases where a serum ferritin or serum iron test was ordered within the last 12 months. Prior serum ferritin results may influence the diagnoses of iron deficiency due to a variety of reasons, including continuation of care to assess the effect of any prior treatments or re-testing to confirm results. Although re-test interval is recommended as 3 months, our criteria was purposefully wide to ensure no potential known-cases were included. Likewise, we excluded patients who had a past iron medication or a past diagnosis for iron deficiency or anaemia at any time.

Presence of inflammation can complicate the diagnosis of iron deficiency, as serum ferritin levels rise under this condition, ${ }^{13}$ and can mask an underlying iron deficiency. Where inflammation is suspected, clinicians may either delay testing for iron deficiency until inflammation is resolved, or order a CRP test, an inflammation marker, and interpret ferritin results in context of the CRP result. ${ }^{14}$ We excluded test results accompanied by high CRP results, as indicated by the pathology provider's report or having a result of $>10 \mathrm{mg} / \mathrm{L}$ to account for possible inflammatory diseases.

Thalassemia can often be mistaken for iron deficiency. We therefore excluded patients who had a known thalassemia diagnosis. Only test results containing both serum iron and serum ferritin results were included.

\section{Statistical analyses}

We set out to determine association between serum iron test results and iron-deficiency diagnoses in the following two population groups: (1) in an iron-deplete population group and (2) in an iron-replete population group. Iron deplete was defined as having low serum ferritin levels: denoted as the serum ferritin result value being $<12 \mu \mathrm{g} / \mathrm{L}$ for children $<5$ years of age and $<15 \mu \mathrm{g} / \mathrm{L}$ for children $\geq 5$ years of age. ${ }^{6}$ A patient was considered as iron replete if their serum ferritin result was either denoted as normal or high by the pathology provider, or had a ferritin result value $>30 \mu \mathrm{g} / \mathrm{L} .{ }^{15}$ Results for other thresholds $(<15 \mu \mathrm{g} / \mathrm{L}$ and $<20 \mu \mathrm{g} / \mathrm{L}$ for iron deplete; $>50 \mu \mathrm{g} / \mathrm{L}$ and $>100 \mu \mathrm{g} / \mathrm{L}$ serum ferritin for iron replete) can be found in the online supplemental file 1 .

In our iron-deplete population group, we tested if having normal serum iron results decreased the odds of a diagnosis; which indicates underdiagnosis. In our ironreplete population group, we tested if having low serum iron results increased the odds of an iron-deficiency diagnosis; which indicates overdiagnosis (figure 1). For the purposes of our study, the term 'diagnosis' comprises a diagnostic record, referral, medication or treatment related to iron deficiency; identified as described under 


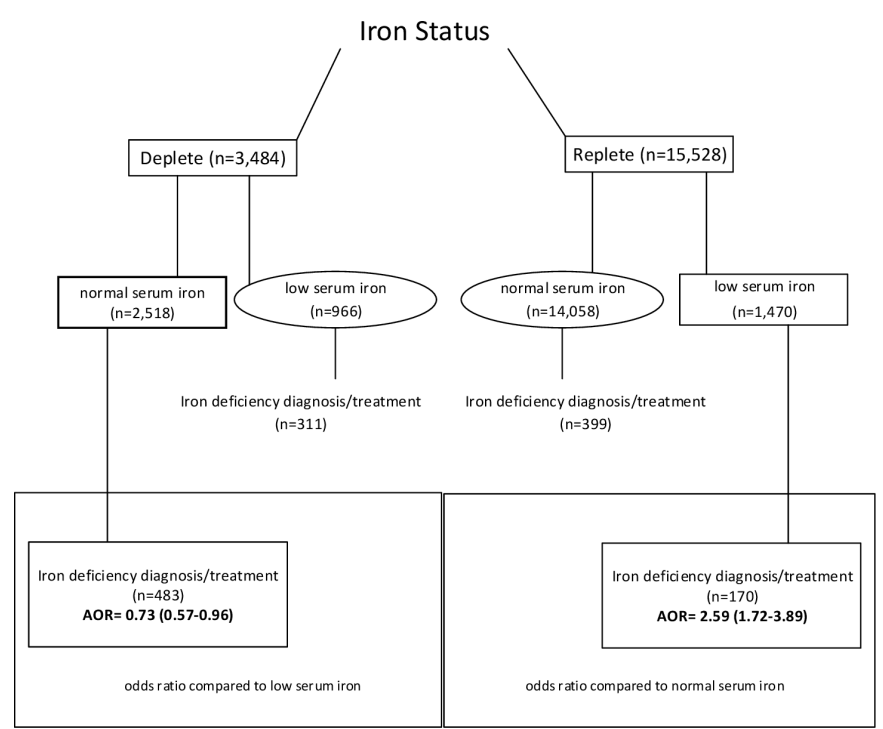

Figure 1 Characteristics of the study population. Iron deplete was defined as having a serum ferritin result value of $<12 \mu \mathrm{g} / \mathrm{L}$, while iron replete was defined as having a serum ferritin result value of $>30 \mu \mathrm{g} / \mathrm{L}$. AOR and related $95 \% \mathrm{Cl}$ present the odds of an iron-deficiency diagnosis in the irondeplete population associated with normal serum iron results, and in the iron-replete population, diagnosis associated with low serum iron results. AOR, adjusted OR.

the study population and variables heading within the Methods section.

Generalised estimating equations were formed to model the probability of an iron-deficiency diagnosis associated with serum iron test results, fitted as a binomial distribution with a logit link function and robust SE estimations. Clusters by general practice and patient were formed, with exchangeable within group correlation. The model was used to report ORs, p values and 95\% CIs with iron-deficiency diagnosis as the binary outcome variable (diagnosed and not diagnosed). Serum iron test result was our main exposure variable of interest, as a binary variable (pathology provider noted low serum iron levels and pathology provider noted normal or high serum iron levels). Covariates included in our model were age (continuous), sex (binary), serum ferritin result value (continuous), mean corpuscular volume, mean corpuscular haemoglobin and mean corpuscular haemoglobin concentration values reported by pathology provider (binary variable as low result value, normal or high result value), as well as red cell distribution width (binary variable as high result value, normal or low result value) and TSAT (binary variable as: low result value, normal or high result value). We also included anaemia status as a covariate, identified as having a haemoglobin concentration $<12 \mathrm{~g} / \mathrm{dL}(120 \mathrm{~g} / \mathrm{L})$ for females and $13 \mathrm{~g} / \mathrm{dL}$ $(130 \mathrm{~g} / \mathrm{L})$ for males. Our covariates were selected due to their relevance to the diagnosis of iron deficiency. Female sex and younger age have high prevalence of iron deficiency. ${ }^{1617}$ Red cell indices are relevant to the diagnosis of iron-deficiency anaemia, and serum transferrin is ordered alongside serum ferritin and can be an indicator of tissue
Table 1 Associations between normal iron test results and iron-deficiency diagnosis for the iron-deplete population

\begin{tabular}{ll}
\hline OR (95\% Cl ; p value) & \\
\hline Normal serum iron result & $0.46(0.40$ to $0.52 ;<0.001)$ \\
\hline Adjusted OR (95\% CI ; p value) & \\
\hline Normal serum iron result & $0.73(0.57$ to $0.96 ; 0.022)$ \\
\hline Female sex & $1.41(1.09$ to $1.81 ; 0.009)$ \\
Increasing age & $1.01(0.99$ to $1.03 ; 0.526)$ \\
\hline Anaemia & $1.19(0.96$ to $1.48 ; 0.115)$ \\
\hline Low MCV & $1.22(0.92$ to $1.63 ; 0.172)$ \\
Low MCH & $1.15(0.89$ to $1.49 ; 0.272)$ \\
Low MCHC & $0.77(0.62$ to $0.97 ; 0.027)$ \\
High RDW & $1.23(0.92$ to $1.63 ; 0.158)$ \\
Low TSAT & $1.03(0.81$ to $1.32 ; 0.797)$ \\
Increasing serum ferritin value & $0.91(0.88$ to $0.94 ;<0.001)$
\end{tabular}

ORs present the binary association between the indicated variable and iron-deficiency diagnosis. Adjusted ORs present the multivariate association between serum iron result and diagnosis, with all covariates included in the model.

$\mathrm{MCH}$, mean corpuscular haemoglobin; $\mathrm{MCHC}$, mean corpuscular haemoglobin concentration; MCV, mean corpuscular volume; RDW, red cell distribution width; TSAT, transferrin saturation.

iron absorption (if interpreted in consideration of serum iron levels), although its use in the diagnosis of iron deficiency is not recommended. ${ }^{6}$ All statistical analyses were conducted using Stata MP V.16.0 (StataCorp, TX, USA).

\section{Patient and public involvement}

Patients were not involved in this study.

\section{RESULTS}

A total of 17929 test results from 14187 patients were included in our analyses. Females accounted for $59.7 \%$ of the test results, and the median age was 14 years (10-17 years).

A total of 3484 test results with low serum ferritin results (ie, iron deplete) were identified (figure 1). There were 2518 test results with normal serum iron, of which 483 were diagnosed with iron deficiency. Our analysis revealed a decrease in the odds of an iron-deficiency diagnosis by 0.73 (95\% CI 0.56 to 0.96 ; p value 0.022 ) when serum iron results were normal (table 1). Having a low MCHC result (adjusted OR (AOR) 0.77 ; $95 \%$ CI 0.62 to 0.97 ; p value 0.027 ), and increasing serum ferritin result value (AOR $0.91 ; 95 \%$ CI 0.88 to 0.94 ; $\mathrm{p}$ value 0.001 ) also decreased the odds of a diagnosis, and female sex (AOR $1.40 ; 95 \%$ CI 1.09 to 1.81 ; p value 0.009 ) increased the odds of a diagnosis.

A total of 15528 test results had normal or high serum ferritin levels (ie, iron replete). Low serum iron results accompanied 1470 of these tests, with a further 170 iron-deficiency diagnoses recorded among these tests (figure 1). We observed a statistically significant increase 
Table 2 Associations between low iron test results and iron-deficiency diagnosis for the iron-replete population

\section{OR ( $95 \% \mathrm{Cl}$; p value)}

Low serum iron result

4.57 (3.94 to $5.30 ;<0.001)$

\begin{tabular}{ll}
\hline \multicolumn{2}{l}{ Adjusted OR $(95 \% \mathbf{C l} ; \mathbf{p}$ value) } \\
\hline Low serum iron result & $2.59(1.72$ to $3.89 ;<0.001)$ \\
Female sex & $1.62(1.34$ to $1.96 ;<0.001)$ \\
Increasing age & $1.04(1.01$ to $1.06 ; 0.001)$ \\
Anaemia & $1.81(1.40$ to $2.35 ;<0.001)$ \\
Low MCV & $0.76(0.36$ to $1.63 ; 0.486)$ \\
Low MCH & $0.94(0.58$ to $1.51 ; 0.792)$ \\
Low MCHC & $0.94(0.64$ to $1.39 ; 0.757)$ \\
High RDW & $1.79(0.92$ to $3.49 ; 0.087)$ \\
Low TSAT & $1.84(1.23$ to $2.75 ; 0.003)$ \\
Increasing serum ferritin & $1.004(1.002$ to $1.01 ;<0.001)$ \\
value &
\end{tabular}

ORs present the binary association between the indicated variable and iron-deficiency diagnosis. Adjusted ORs present the multivariate association between serum iron result and diagnosis, with all covariates included in the model.

$\mathrm{MCH}$, mean corpuscular haemoglobin; $\mathrm{MCHC}$, mean corpuscular haemoglobin concentration; MCV, mean corpuscular volume; RDW, red cell distribution width; TSAT, transferrin saturation.

in the odds of iron-deficiency diagnosis with low serum iron results by 2.59 (95\% CI 1.72 to 3.89; $p$ value $<0.001$; table 2). Female sex (AOR 1.62; 95\% CI 1.34 to 1.96; p value $<0.001$ ), having anaemia (AOR 1.81; 95\% CI 1.40 to 2.35; p value $<0.001$ ) and having low TSAT (AOR 1.84; $95 \%$ CI 1.23 to 2.75 ; p value 0.003 ) were also associated with an increased odds of diagnosis.

\section{DISCUSSION}

Our study identified both underdiagnosis and overdiagnosis of iron deficiency among children treated in general practice related to the interpretation of serum iron results. Children without iron deficiency were over two times more likely to be erroneously diagnosed (overdiagnosed) when they had low serum iron results. Children with iron deficiency were 0.7 times less likely to be diagnosed (underdiagnosed) when they had normal serum iron levels.

Our study demonstrates serum iron results being erroneously used in the diagnosis of iron deficiency, consequently leading to diagnostic errors. Our results suggest a misconception of the changed role of iron levels in diagnosing iron deficiency; serum iron being confused as the biomarker of iron deficiency where in fact, serum ferritin is. Serum iron tests performed as part of a panel act as a distractor in interpreting the test.

We also observed an increase in the likelihood of an iron deficiency diagnosis with low haemoglobin results. Low haemoglobin levels are indicative of anaemia, and the most common cause of anaemia is iron deficiency. ${ }^{18}$
Based on Croskerry's ${ }^{19}$ study on biases in decision making, our observation may be a result of 'availability and non-availability' bias; defined as a tendency to make a judgement based on a thought coming more readily to mind due to its frequency. Alternatively, it may be a result of 'information distortion bias', defined by Boyle and Purdon ${ }^{20}$ as using information to support of a course of action more so than is warranted by the available information. Anaemia is frequently caused by iron deficiency and may have consequently led to the misdiagnosis. We also observed females being more likely to be diagnosed with iron deficiency. Females are known to have a high incidence of iron deficiency in the general population, predominantly thought to be a result of menses. ${ }^{21}$ Similar to haemoglobin, female sex could also be resulting in bias in interpretation of the iron panel tests due to its stronger association with the disease.

Laboratory testing-related errors are one of the highest contributing factors to diagnostic errors, as demonstrated by Schiff $e t a l \mathrm{~s}^{22}$ study. Schiff $e t a l^{22}$ asked clinicians across the USA to report three cases of diagnostic errors, and describe their cause, significance and frequency. They found that laboratory testing-related errors, including erroneous interpretation of test results, were the highest contributor to diagnostic errors: with $44 \%$ of the errors accounted to it. In Hickner et $a l \mathrm{~s}^{23}$ survey, doctors reported uncertainty in test result interpretation $8.3 \%$ of the time. Some of the reported challenges related to nomenclature of the tests, such as different tests with the same name, or different names for the same test.

In Australia, a Medicare review taskforce was formed to ensure current clinical practice aligned with evidence and best practice. ${ }^{24}$ Iron panel tests were found to be ordered over three times higher than standalone ferritin tests by general practitioners in diagnosing iron deficiency. Our results provide empirical evidence demonstrating the resultant diagnostic errors due to serum iron tests among children. The Medicare taskforce made recommendations to relabel tests in clinically relevant terms, such that a clinician would order 'iron-deficiency studies' containing serum ferritin, rather than the current iron panel test with all iron biomarkers. Such an approach may reduce cognitive errors by removing confounding factors out of the decision-making process. In the diagnosis of iron deficiency, this may reduce burden of disease by ensuring patients with iron deficiency are correctly and timely diagnosed, potentially preventing anaemia and cognitive impairment. Additionally, correct use of serum ferritin to diagnose iron deficiency may reduce the number of patients being misclassified as having iron deficiency based on serum iron results, potentially reducing unnecessary treatment and promoting correct diagnosis by ruling out iron deficiency.

Some limitations should be taken into consideration when interpreting the findings of our study. Our study sample is derived from general practice electronic health records, and the data quality and completeness are therefore largely influenced by general practitioner's 
use of their software. The reason for ordering of iron panel tests is not known, and may have an impact on the diagnosis. Nonetheless, serum ferritin is a test used in checking the iron status of a patient, and low levels, particularly $<12 / 15 \mu \mathrm{g} / \mathrm{L}$ should be treated. Diagnostic records are often not complete and many diagnoses, including iron deficiency, are omitted. Certain diseases may impact the interpretation of serum ferritin and the diagnosis of iron deficiency, including thalassemia and inflammatory diseases. We accounted for a number of these potential confounders, although it is likely that other unknown factors (diseases, clinical investigations and patient history) may have impacted the interpretation of test results and the diagnosis of iron deficiency. Although medication prescriptions are well recorded, iron-deficiency medication can be obtained without prescription; however, the dosage of over the counter iron supplements may not be appropriate for patients with $<12 / 15 \mu \mathrm{g} / \mathrm{L}$ serum ferritin, particularly for paediatric patients. We identified the closest iron deficiencyrelated diagnosis and medication records within a cut-off period of 14 days after the test result as our outcome. It is possible that some records were missed due to this time period, although we expect a majority of patients to return to the practice within this timeframe. Nonetheless, it is also possible that the patient may not return to the clinic at all, particularly if their perceived condition has improved. This has additional consequences on one of our outcome criteria: follow-up within the next 3 months. These limitations may have resulted in an underestimate of our outcome measure. Nonetheless, our study objective did not necessitate all of the potential outcomes to be included in the analysis to reach a meaningful conclusion. A sample size large enough to make a meaningful inference from our sample to the population, and no systematic bias in the outcome measure in one category in relation to the other which may have occurred due to missed cases, was required. Based on our final study sample size and the possibility for a systematic bias between our categories being highly unlikely, we are confident both criteria have been achieved and our conclusion is accurate. Nonetheless, the effect size should be cautiously interpreted, which may have been affected by the missed cases.

Our study also has several strengths. Our outcome was identified through several criteria, in line with the RECORD-PE guidelines: a diagnostic record, medication record, specialist referral or follow-up treatment. Our iron-deplete population, having $<12 / 15 \mu \mathrm{g} / \mathrm{L}$ serum ferritin, requires treatment with medication and follow-up..$^{25}$ We therefore believe that most patients who are diagnosed and are being treated would be captured with our criteria.

To the best of our knowledge, our study is the first to quantitatively demonstrate the interpretation biases of serum iron test results in general practice, and the resultant error in diagnosing iron deficiency. Iron panel tests are ordered in a similar fashion worldwide in diagnosing iron deficiency, with all four tests being included. Although our results are from Australian general practices within the state of Victoria, it is possible that a similar diagnostic error is occurring in other states/territories and countries where iron panel tests are ordered in the investigation of iron deficiency instead of standalone serum ferritin.

\section{CONCLUSIONS}

Our study demonstrates cognitive biases in diagnosing iron deficiency in children, which is related to serum iron results and augmented by abnormal haemoglobin test results and female sex. Serum iron tests have consequences for both overdiagnosis and underdiagnosis, as it appears to lead general practitioners to overlook serum ferritin tests results. In diagnosing iron deficiency, serum iron levels are no longer indicated by clinical guidelines and should not form a basis in establishing iron-deficiency status of patients.

\section{Author affiliations}

${ }^{1}$ Faculty of Medicine and Health Sciences, Macquarie University, Sydney, New South Wales, Australia

${ }^{2}$ Gippsland Primary Health Network, Traralgon, Victoria, Australia

${ }^{3}$ Outcome Health, Burwood, Victoria, Australia

${ }^{4}$ Department of Paediatrics, The University of Melbourne, Melbourne, Victoria, Australia

${ }^{5}$ Murdoch Childrens Research Institute, Parkville, Victoria, Australia

${ }^{6}$ Paediatrics, University of Melbourne, Parkville, Victoria, Australia

\section{Twitter A Georgiou @AGeorgiouMQ}

Contributors GS conceptualised the study, carried out the statistical analysis, drafted the initial manuscript, and reviewed and revised the manuscript. PM and VI conceptualised the study, contributed to the drafting of the initial manuscript, provided expertise and critically reviewed the manuscript for important intellectual content. LL provided expertise on the statistical analysis, contributed to the design of the study, and reviewed and revised the manuscript for important intellectual content. AML and CP developed the data collection instruments, collected data, contributed to the interpretation of the data, and reviewed and revised the manuscript. JW, AG, EW and DA contributed to the design of the study, critically reviewed the manuscript for important intellectual content and revised the manuscript. All authors approved the final manuscript as submitted and agree to be accountable for all aspects of the work.

Funding This study was supported by funding from the Quality Use of Pathology Program, Department of Health, Government of Australia (4-2QFVW4M).

Competing interests None declared.

Patient consent for publication Not required.

Ethics approval Approval to use the de-identified general practice electronic health record data in research was obtained by the data custodians, Outcome Health, from the RACGP National Research and Evaluation Ethics Committee (NREEC 17-008). Ethics approval for the use of the de-identified data was obtained from Macquarie University Human Research Ethics Committee (5201700872).

Provenance and peer review Not commissioned; externally peer reviewed.

Data availability statement Data are available upon reasonable request. Deidentified individual participant data, and ethics approval documents for the collection of data by the data custodians Outcome Health can be made available to researchers who provide a methodologically sound proposal for use in achieving the goals of their approved proposal, on condition of approval of the primary health networks on whose behalf the data is being collected for. Proposals should be submitted to Outcome Health at admin@outcomehealth.org.au.

Supplemental material This content has been supplied by the author(s). It has not been vetted by BMJ Publishing Group Limited (BMJ) and may not have been 
peer-reviewed. Any opinions or recommendations discussed are solely those of the author(s) and are not endorsed by BMJ. BMJ disclaims all liability and responsibility arising from any reliance placed on the content. Where the content includes any translated material, BMJ does not warrant the accuracy and reliability of the translations (including but not limited to local regulations, clinical guidelines, terminology, drug names and drug dosages), and is not responsible for any error and/or omissions arising from translation and adaptation or otherwise.

Open access This is an open access article distributed in accordance with the Creative Commons Attribution Non Commercial (CC BY-NC 4.0) license, which permits others to distribute, remix, adapt, build upon this work non-commercially, and license their derivative works on different terms, provided the original work is properly cited, appropriate credit is given, any changes made indicated, and the use is non-commercial. See: http://creativecommons.org/licenses/by-nc/4.0/.

\section{ORCID iDs}

Gorkem Sezgin http://orcid.org/0000-0001-6429-7745

A Georgiou http://orcid.org/0000-0002-7619-3668

\section{REFERENCES}

1 Kassebaum NJ, Jasrasaria R, Naghavi M, et al. A systematic analysis of global anemia burden from 1990 to 2010. Blood 2014;123:615-24.

2 Beard JL. Why iron deficiency is important in infant development. $J$ Nutr 2008;138:2534-6.

3 Camaschella $\mathrm{C}$. New insights into iron deficiency and iron deficiency anemia. Blood Rev 2017;31:225-33.

4 Soppi ET. Iron deficiency without anemia - a clinical challenge. Clin Case Rep 2018;6:1082-6.

5 Pasricha S-RS, Flecknoe-Brown SC, Allen KJ, et al. Diagnosis and management of iron deficiency anaemia: a clinical update. Med $J$ Aust 2010;193:525-32.

6 World Health Organization (WHO). WHO guideline on use of ferritin concentrations to assess iron status in individuals and populations. Geneva: World Health Organization (WHO), 2020.

7 Laposata M. Errors in clinical laboratory test selection and result interpretation: commonly unrecognized mistakes as a cause of poor patient outcome. Diagnosis 2014;1:85-7.

8 Plebani M. Diagnostic errors and laboratory medicine - causes and strategies. EJIFCC 2015;26:7-14.

9 Pearce C, McLeod A, Rinehart N, et al. What a comprehensive, integrated data strategy looks like: the population level analysis and reporting (polar) program. Stud Health Technol Inform 2019;264:303-7.
10 Langan SM, Schmidt SA, Wing K, et al. The reporting of studies conducted using observational routinely collected health data statement for pharmacoepidemiology (RECORD-PE). BMJ 2018;363:k3532.

11 Stram M, Gigliotti T, Hartman D, et al. Logical observation identifiers names and codes for Laboratorians. Arch Pathol Lab Med 2020;144:229-39.

12 Côté RA, Robboy S. Progress in medical information management. Systematized Nomenclature of medicine (SNOMED). JAMA 1980;243:756-62.

13 Kell DB, Pretorius E. Serum ferritin is an important inflammatory disease marker, as it is mainly a leakage product from damaged cells. Metallomics 2014;6:748-73.

14 Cook JD. Diagnosis and management of iron-deficiency anaemia. Best Pract Res Clin Haematol 2005;18:319-32.

15 WHO. Serum ferritin concentrations for the assessment of iron status and iron deficiency in populations, 2020. Available: https://www.who. int/vmnis/indicators/serum_ferritin.pdf

16 Percy L, Mansour D, Fraser I. Iron deficiency and iron deficiency anaemia in women. Best Pract Res Clin Obstet Gynaecol 2017:40:55-67.

17 Parkin PC, Borkhoff CM. Practical tips for paediatricians: assessment and management of young children with iron deficiency. Paediatr Child Health 2018;23:433-4

18 World Health Organization, Centers for Disease Control and Prevention (U.S.), De Benoist B. Worldwide prevalence of anaemia 1993-2005 of: WHO global database of anaemia. Geneva: World Health Organization, 2008. http://whqlibdoc.who.int/publications/ 2008/9789241596657_eng.pdf

19 Croskerry P. Achieving quality in clinical decision making: cognitive strategies and detection of bias. Acad Emerg Med 2002;9:1184-204.

20 Boyle PJ, Purdon M. The information distortion bias: implications for medical decisions. Med Educ 2019;53:1077-86.

21 Rushton $\mathrm{DH}$, Barth $\mathrm{JH}$. What is the evidence for gender differences in ferritin and haemoglobin? Crit Rev Oncol Hematol 2010;73:1-9.

22 Schiff GD, Hasan O, Kim S, et al. Diagnostic error in medicine: analysis of 583 physician-reported errors. Arch Intern Med 2009;169:1881-7.

23 Hickner J, Thompson PJ, Wilkinson T, et al. Primary care physicians' challenges in ordering clinical laboratory tests and interpreting results. J Am Board Fam Med 2014;27:268-74.

24 MBS Review Taskforce. Diagnostic medicine clinical Committee final report, 2020. Available: https://www1.health.gov.au/internet/main/ publishing.nsf/Content/mbs-review-2018-taskforce-reports-cp/\$File/ Diagnostic-Medicine-Clinical-Committee-Final\%20Report.pdf

25 Ozdemir N. Iron deficiency anemia from diagnosis to treatment in children. Turk Arch Ped 2015;50:11-19. 